\title{
A New CMOS OP-AMP Design with an Improved Adaptive biasing circuitry
}

\author{
HATIM AMEZIANE ${ }^{1}$, KAMAL ZARED ${ }^{2}$, AND HASSAN QJIDAA $^{3}$ \\ 1,2,3 Physics Department- CED-ST, LESSI, Faculty of Sciences \\ Sidi Mohamed Ben Abdellah University \\ BP 1796, $30003 \mathrm{Fez}$ \\ MOROCCO
}

\begin{abstract}
This paper sets out a new technique for designing an operational amplifier (OP-AMP) using tanner EDA 1um FDSOI CMOS Technology. Fully Depleted Silicon on Insulator used for building integrated circuits to support the temperature changes, the proposed OP-AMP operates at $3.75 \mathrm{~V}$ power supply and $70 \mathrm{uA}$ bias current using the proposed Adaptive Biasing Circuitry $(\mathrm{ABC})$, which its devices operate at the weak inversion to allow low power dissipation of $0.62 \mathrm{~mW}$. The $0.064 \mathrm{us}$ settling time and $37.016 \mathrm{~V} / \mu \mathrm{s}$ slew rate parameters improved by the $\mathrm{ABC}$ technique, reducing the power dissipation by operating the $\mathrm{ABC}$ devices in weak inversion. The phase margin is more than 100 degrees for the $\mathrm{DC}$ gain of $13.97 \mathrm{~dB}$, which is a reasonable margin when temperature range increases.
\end{abstract}

Key-Words: - Analog Design, OP-AMP, Amplifier, CMOS, Low Power, Settling Time, Weak Inversion.

Received: April 29, 2020. Revised: September 30, 2020. Accepted: November 1, 2020. Published: November 28, 2020.

\section{Introduction}

The operational amplifier (OP-AMP) is one of the essential analog circuits for all modern instrumentations such as aerospace and industrial instrumentations, where the output voltage changes quickly from one level to another is mandatory. The parameters of low power dissipation, high temperature, high speed, excellent power supply rejection, and high gain are becoming more important in miniature analog circuits to provide the significant results; these results could be a concern in high-resolution systems [1]. Furthermore, the OPAMP is one of the fundamental analog blocks of every signal processing tasks, either in weak signal amplifications or complex processing applications in mixed-signal domain such as portables application and a among various audio/video applications [2]. The OP-AMP is so denominated because its original purpose was to provide mathematical operations such as subtraction, multiplication, integration and differentiation, etc. It can be used in instrumentation, scale changing, and a great variety control system [3].

An OP-AMP is essential to deliver the appropriate parameters to match the requirements of various analog converters. In Low dropout (LDO) regulators and switching converters, an OP-AMP used as an error amplifier to increase the control accuracy of the power transistor [4] [5]. In the Analog/Digital Converters (ADC), the op amp can be used as a buffer to provide the appropriate offset and gain to match the output signal to the input range [6]. Choosing the correct amplifier for a specific application needs to have a precise design. Therefore, it is necessary to develop a design that helps providing the desired parameters such as the (Width/Length) ratio, as well as choosing the best process technology [7]. These inspire developing a special biasing technique, which increases the OPAMP performances.

At a high temperature operation, the System-OnChip (SOC) circuits implemented in SOI CMOS are characterized by many benefits including: subthreshold region or weak inversion and power reduction, and high transconductance (gm). The proposed Silicon on Insulator (SOI) 1um technology in the SoC solutions is being as the next node in the analog design of low power, the benefits of this technology such as minimizing the input voltage and reducing the gate capacitances led to minimize the power dissipation, which is important for the embedded applications. The transistor canal in SOI technology is invented to separate from the bulk by an insulation layer to reduce the gate capacitors thus turns the SoC faster and reducing overall noise. 
Moreover, the benefits of high-energy efficiency and low power in weak inversion operation region can also provide the highest transconductance gm. These make SOI technology an ideal choice for analog circuits and low power application. Transconductance Amplifiers in SOI technology process with Low power dissipation have been obtained in previous works $[8,9]$.

Taking into consideration high-temperature design, we will focus on the design of an OP-AMP in this present work, which is a critical analog block in any embedded system. We will discuss the design, performance and simulation results of the OP-AMP and its bias circuit (ABC).

\section{Design Considerations of The Proposed Op-Amp}

The conceptual schematic of the proposed OPAMP is shown in figure 2, which contains the bias circuit to make the OP-AMP self-biased. Indeed, the main grant of the present OP-AMP in the embedded systems is that it has a low power dissipation of the output stage, as well as a significant phase margin that allows working in very high temperature with guaranteed stability in a wide frequency range.

The OP-AMP architecture is chosen in this work since it has been proven to have a good stability and a high performance at high temperatures. Moreover, the layout of the proposed OP-AMP presented in figure 10 has been designed in compliance with all layout design rules and transistor matching standards in order to integrate it in a SoC.

The operating in weak inversion of MOS transistors in this OP-AMP allows reducing the power consumption of the global system. The current $I_{D S}$ through the MOS transistor channel operating in sub-threshold region based on the channel diffusion current.

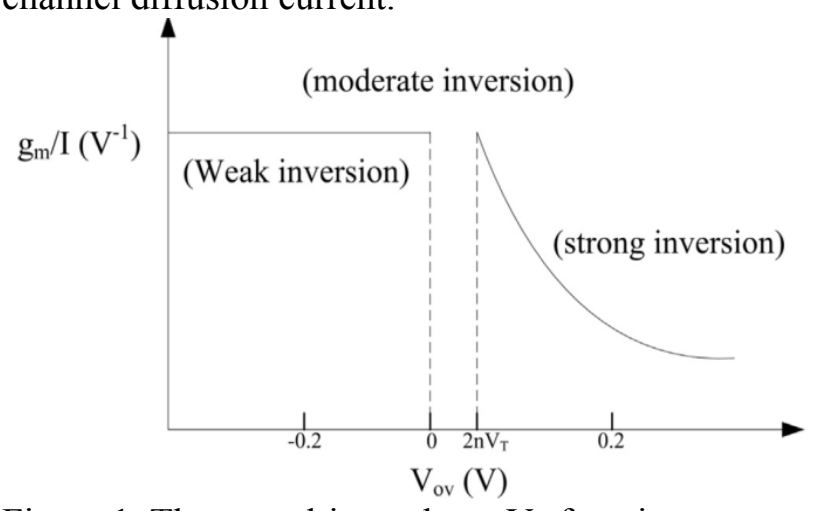

Figure 1. The over drive voltage Vs function regimes.
When VDS $\geq 3 \mathrm{kT} / \mathrm{q}$, the transistor will be saturated and $I_{D S}$ can be given by:

$$
I_{D S}=\frac{1}{2} g_{m-S a t}\left(V_{G S}-V_{T H}\right)=\frac{1}{2} g_{m-S a t} V_{O V}
$$

While in sub-threshold region can be expressed by:

$$
I_{D S}=g_{m-S u b} \eta V_{T}
$$

Where gm-sub and gm-sat are the transconductance in sub-threshold and saturation regions respectively current, $\mathrm{n}$ is the factor of slope in sub-threshold region which can be defined as $1+$ $\mathrm{gmb} / \mathrm{gm}$.

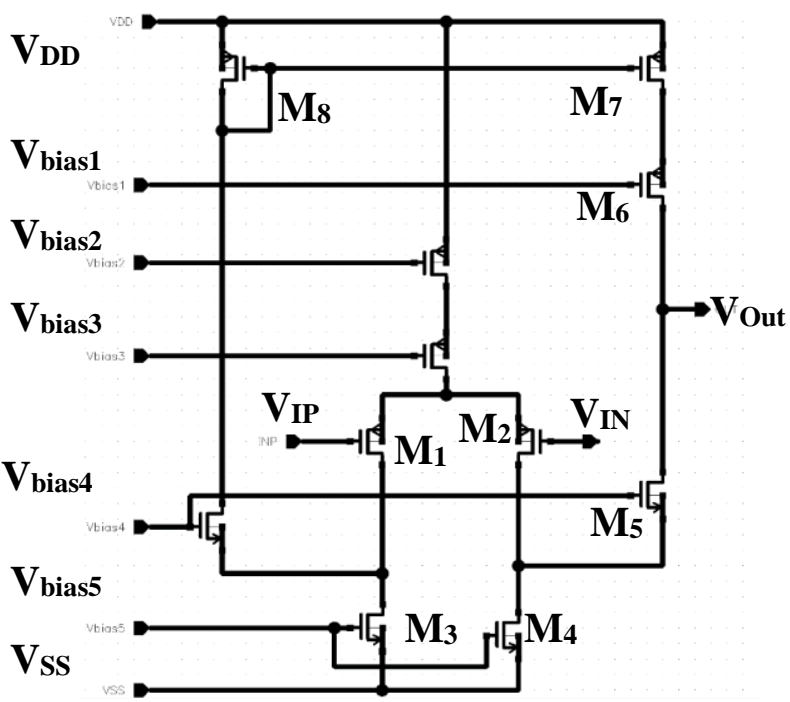

(a)

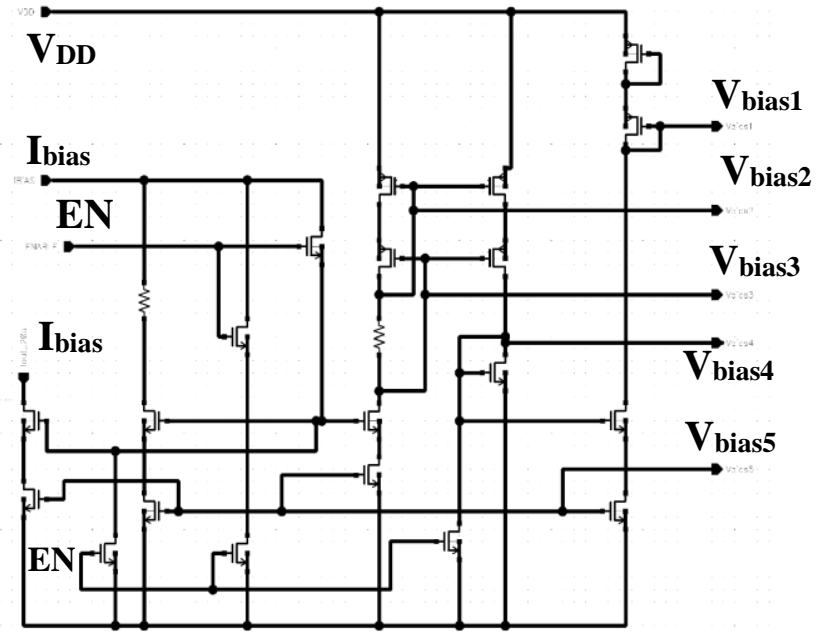

(b)

Figure 2. (a) Schematic of the proposed OP-AMP (b) the Adaptive Biasing Circuitry (ABC)

Since threshold voltage $\mathrm{V}_{\mathrm{TH}}=\mathrm{V}_{\mathrm{TO}}-(\mathrm{n}-1)$ VBS, $\mathrm{V}_{\mathrm{OV}}$ is the over drive voltage and the other symbols 
have their usual meanings. To make sure the Adaptive Biasing Circuitry (ABC) works in subthreshold region instead of the strong inversion function in order to have a high transconductance, VGS should be less significant than $\mathrm{V}_{\mathrm{OV}}+\mathrm{V}_{\mathrm{TH}}$ as shown in fig 1:

In the following subsections, the composition of the proposed OP-AMP will be discussed and simulations will show that an excellent settling time is obtained with a significant save in power consumption by implementing the folded cascode biasing circuit, providing a good combination of gain and minimum on chip size.

\subsection{The Proposed Adaptive Biasing Circuitry (ABC)}

The implementation of the bias circuit shown in fig.2 (b) allows increasing driving capability with an increase of the OP-AMP complexity. Thus, short slew rate and high-speed driving capabilities can be completed during the transient operation. Moreover, to adapt the proposed OP-AMP to function in the desired high temperature, we have adopted a biasing circuit in order to generate a constant transconductance gm of each device, which affects the DC gain, and stability of the OP-AMP.

As shown in [10] a classic bias circuit was proposed to have constant gm. Neglecting the body effects, the gm is given by:

$$
g_{m}=\sqrt{2 \mu_{0} C_{o x} \frac{W}{L}} I_{D S}
$$

\subsection{The proposed OP-AMP design}

The schematic diagram of the proposed OP-AMP is shown in Fig.2 (a). It consists of cross-coupled pairs P-channel (M1-M2) with the two series current generator (M10-M11), a multi-current comparators (M3;M4), (M5;M9), and a current mirror (M7;M8), controlled ultimately by the $\mathrm{ABC}$ circuit in order to determine the quiescent current and minimize the power dissipation, as well as the architecture is designed to minimize the mismatching, providing overall a simple design. Indeed, when a transient input voltage step applied, the currents Ids through the transistors (M1- M2) increase and decrease proportionally. Considering $\Delta \mathrm{V}+$ and $\Delta \mathrm{V}-$, the positive and the negative input steps amplitudes, the drain current of M6 will increase and decrease to switch ON or OFF the transistor M5, while its gate can be reached the input voltage. As shown in [11] ISR, the drain current of M5 and represents the

contribution to the amplifier SR (slew-rate) and can be expressed by:

$$
S R=\frac{\Delta \mathrm{V}_{ \pm}}{d t} \cong \frac{\mathrm{I}_{\mathrm{SR}}}{\mathrm{C}_{\mathrm{L}}}
$$

The Slew-rate enhancement depends on the large load capacitor, this capacitor pushs the dominant pole into the high frequencies which reduces the $\mathrm{AC}$ response prformance [12]. In this proposed OP$\mathrm{AMP}$, the $\mathrm{ABC}$ circuit is used to improve the SR and enable low-voltage operation consuming much less power and driving a 5 (pf) load capacitor.

\subsection{Frequency analysis of the proposed OP- AMP}

Since the biasing circuit Fig.2. (b) provides five reference voltages (constant voltages), only the equivalent small signal diagram of Fig.2. (a) is shown and all reference voltages are connected to the ground. To obtain the gain equation, the small signal model of the proposed OP-AMP shown in Figure 3 is needed: $\frac{V_{\text {out }}}{V_{\text {id }}}$

The differential gain equation is given by: $A_{d}=$

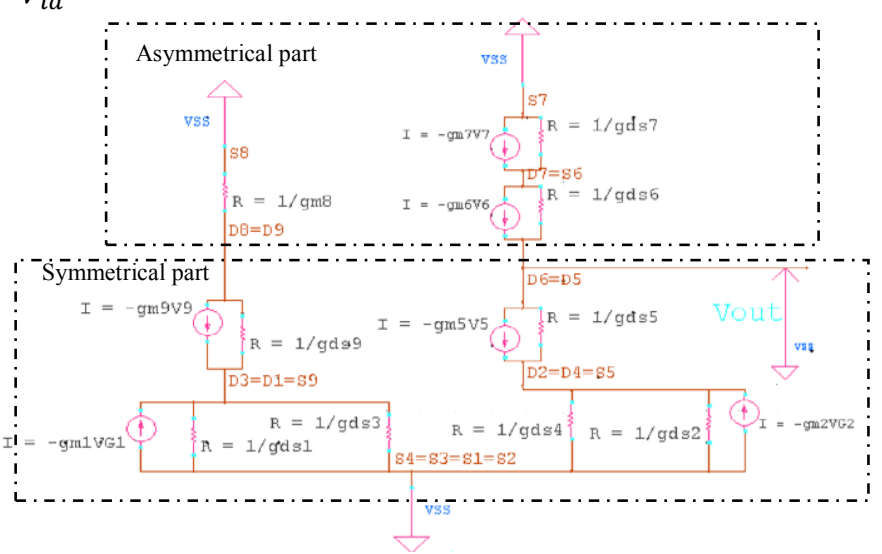

Figure 3. Small signal model of the proposed OP-AMP

(a)



Figure 4. Small signal model of the proposed OP-AMP: (a) and (b) Thevenin presentations of the proposed OP-AMP 
The symmetrical part will be replaced by a generator (Vth) and a Thevenin resistor (Rth), the following diagram is then obtained: Fig.4 (a) and (b). The equivalent scheme for the calculation of Vth (symmetrical part operating in differential mode), V_s1,2 $\approx 0$ will be as follows:

$$
R_{t h}=2\left(\frac{1}{g_{D S 9}}+\frac{1}{g_{D S 1}+g_{D S 3}}\right)
$$

$V_{t h}=2 V_{D 9}=$

$\frac{\left[-\frac{g_{m 9,5} * g_{m 1,2}}{g_{D S 2,1} g_{D S 4,3}}\right]\left[r_{D S 5,9}\left(r_{D S 1,2} / / r_{D S 3,4}\right)\right]}{\left[r_{D S 5,9}+\left(r_{D S 1,2} / / r_{D S 3,4}\right)\right]} V_{I d}$

$$
\begin{gathered}
A_{d}=\frac{V_{o u t}}{V_{i d}}= \\
\frac{g_{m 9,5} * g_{m 1,2} * r_{d s 9,5}\left(r_{d s 1,2} / / r_{d s 3,4}\right)}{\left(g_{D S 2,1}+g_{D S 4,3}\right)\left(r_{d s 9,5}+\left(r_{d s 1,2} / / r_{d s 3,4}\right)\right)}
\end{gathered}
$$

\section{3 simulation results}

The circuit implementation of the proposed OPAMP is implemented in 1um FD-SOI CMOS process. All the simulations that simulated with $(5 \mathrm{pF})$ load mentioned in Table 1 demonstrate that the proposed amplifier has achieved high performances, such as a fast transient response, low power dissipation and a good phase margin. To allow a high GBW (Gain Bandwidth), the ABC circuit should be as low as possible since the UGF of the OP-AMP proposed is stronger dependent on temperature compared to the gain DC.

\subsection{Simulation AC response}

$\mathrm{AC}$ analysis allows us to characterize the cell performance in order to determine the parameters of the open loop gain, the phase margin, CMRR and PSRR. AC analysis is useful to analyze the effect of noise and to determine the distortion characteristics of the circuit. Figure 5 illustrates the gain and phase of the open-loop op amp. As shown in fig.5, the DC gain is $13.97 \mathrm{~dB}$ while the phase margin is 101.62 deg.

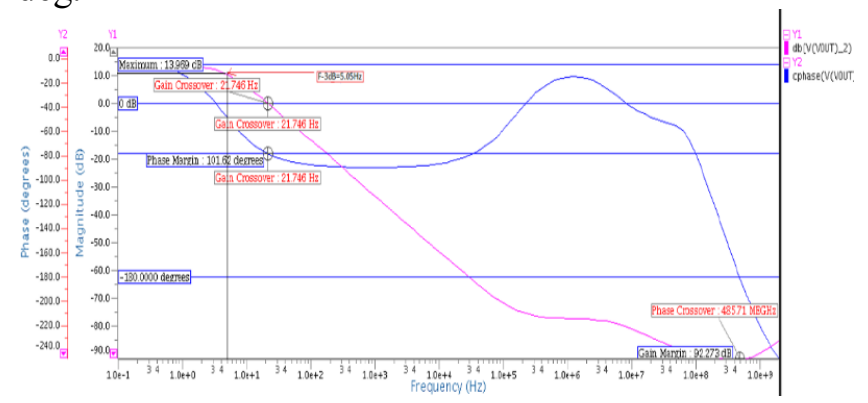

Figure 5. AC response of the proposed OP-AMP

\section{2 transient simulation}

The transient simulation of the amplifier connected as a follower and supplied as an input in the form of a square-wave signal is shown in Figure 6 , which is obtained from the Eldo software.

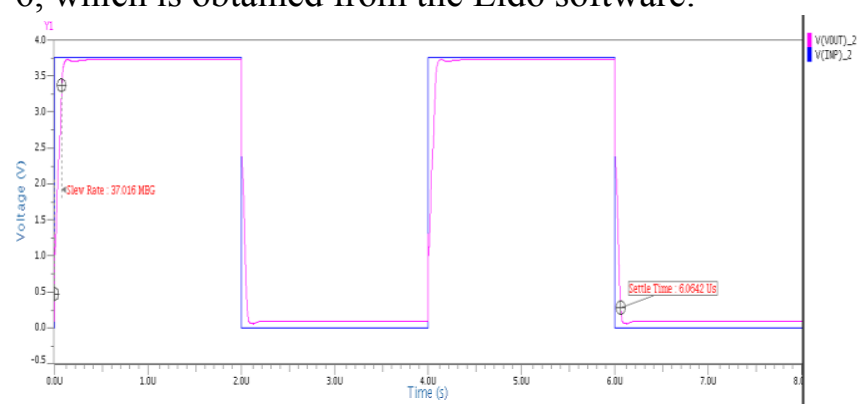

Figure 6. Transient response of the proposed OPAMP

This analysis allows us to validate the stability of the system by ensuring that the output does not oscillate and that it follows the input. The Slew Rate can be defined as the output signal sweep rate, SR given by the expression (4): According to the curve the settling time (ST) and the Slew Rate (SR) are respectively given by $\mathrm{ST}=0.064 \mu \mathrm{s}, \mathrm{SR}=37.016$ MG V/s $=37.016 \mathrm{~V} / \mu \mathrm{s}$.

\subsection{Analysis}

The DC analysis is useful for determining the steady-state point (or bias point) of the circuit, the input or output resistance of the circuit, and the sensitivities of the output variables as a function of circuit parameters. First, we start with the simulation of the biasing circuitry in Figure 2 (b), which generates five reference voltages: Vbias1, Vbias2, Vbias3, Vbias4 and Vbias5. The simulation result shown in Figure 7:

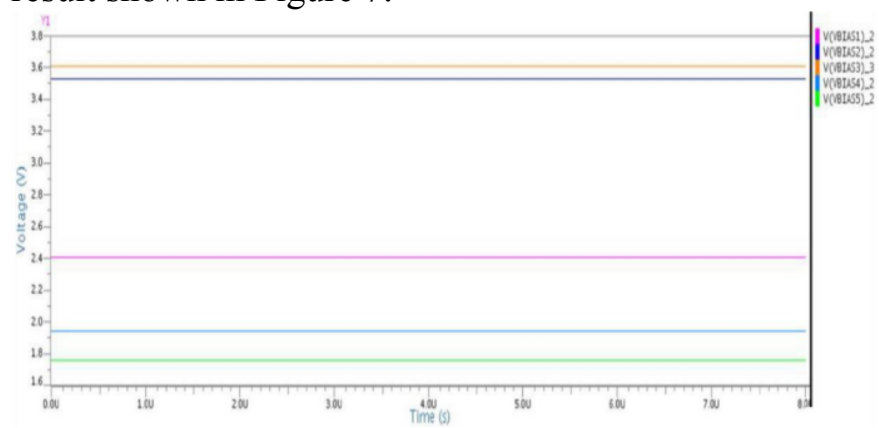

Figure 7. Simulation du circuit de polarisation

\subsection{CMRR Simulation}

The CMRR (Common Mode Rejection Ratio) of an OP-AMP is calculated by multiplying the common mode gain of the differential input stage by the gain of the second stage (the output stage) and 
dividing the open-loop gain on this product CMRR $=$ Ad $/ A c m$. The higher CMRR value, the less sensitive to interferences of the amplifier. In this case the simulation prove that the $\mathrm{CMRR}=41.12 \mathrm{~dB}$ As shown in fig. 8

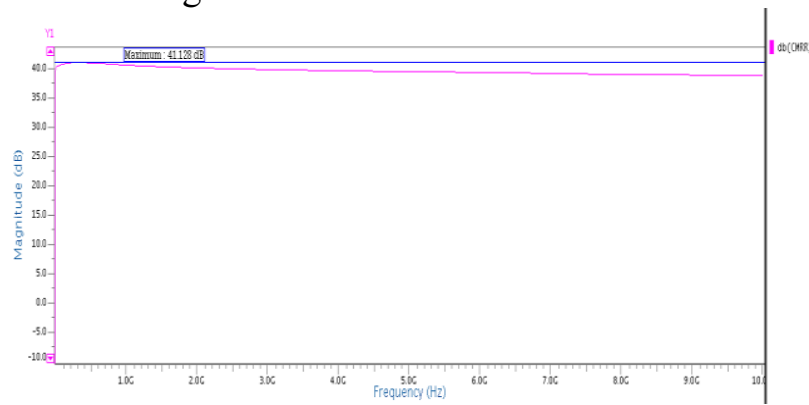

Figure 8. CMRR Magnitude of the proposed OPAMP

\subsection{PSRR simulation}

The PSRR is defined as the variation ratio of the supply voltage to the output voltage of the OPAMP. The PSRR+ is the effect of the VDD variation on the output voltage, given by the following formula: PSRR $+=\Delta V d d /$ Vout. The PSRR- is the effect of VSS variation on the output voltage, given by the following formula: PSRR-= $\triangle V s S /$ Vout. The simulation result of the rejection rate of the supply voltage is shown in the following figure :

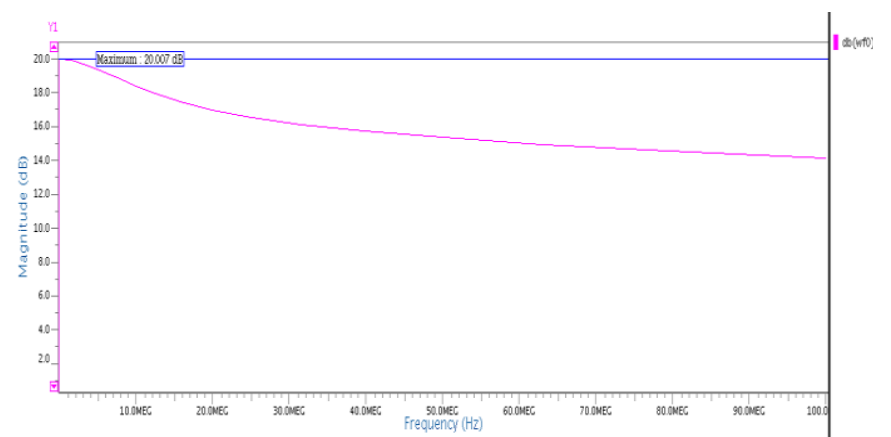

(a)

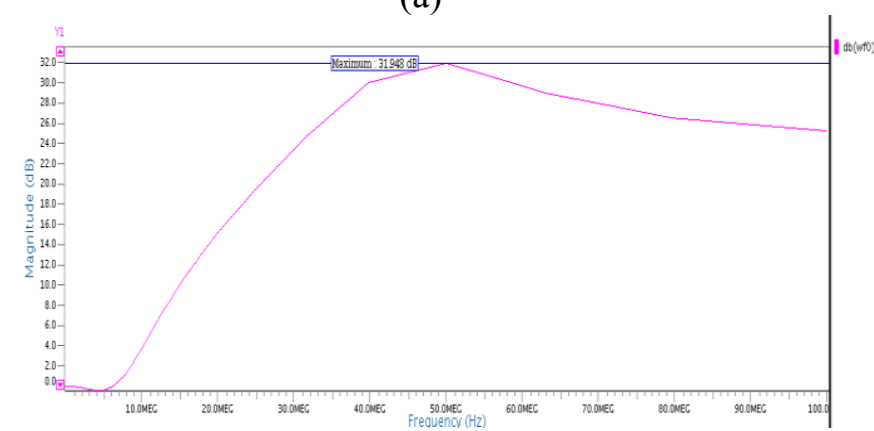

(b)

Figure 9. Power Supply Rejection Ratio Simulation (a) PSRR+ (b) PSRR-

\subsection{Full-on-Chip Layout of the proposed op- amp}

The layout of the OP-AMP circuit is shown in Fig. 10 with an optimized chip size of $0.0313 \mathrm{~mm}^{2}$ $(182 \mu \mathrm{m}, 172 \mu \mathrm{m})$.



Figure 10. Layout of the proposed OP-AMP, including the $\mathrm{ABC}$ circuit

Table 1. Performance of the proposed op-amp and comparison with other works

\begin{tabular}{lccc} 
Parameters & {$[7]$} & {$[11]$} & This work \\
\hline Technology process & CMOS $1.8 \mu \mathrm{m}$ & CMOS $0.35 \mu \mathrm{n}$ & SOI $1 \mu \mathrm{m}$ \\
Input Voltage (V) & 1 & 3 & 3.75 \\
Temperature $\left({ }^{\circ} \mathbf{C}\right)$ & 100 & -10 to 85 & -55 to 225 \\
Biasing Current (uA) & N/A & 1.6 & 7 \\
Load capacitor $(\mathbf{p F})$ & N/A & 1000 & 5 \\
DC Gain(dB) & 67.81 & N/A & 13.97 \\
Phase margin $($ Deg) & 45.9 & 53 & 101.62 \\
Gain Margin(dB) & 67.81 & 91 & 92.27 \\
Slew Rate(V/us) & 0.052 & $6.5 / 8.1$ & 37.01 \\
ICMR+, ICMR- $(\mathbf{V})$ & {$[0 ; 0.854]$} & N/A & {$[0.22 ; 3.6]$} \\
Settling time( $\boldsymbol{\mu}$ s) & N/A & 1.1 & $0.064 \mathrm{us}$ \\
CMRR $(\mathbf{d B})$ & $104.45 @ 10 \mathrm{hz}$ & N/A & 41.12 \\
PSRR-, PSRR+ (dB) & N/A & N/A & $20.07 ; 31.94$ \\
Offset $(\mathbf{m V})$ & N/A & N/A & 0.5 \\
Power Consumption & $7.243 \mathrm{uW}$ & N/A & $0.62 \mathrm{~mW}$ \\
\hline
\end{tabular}

\section{Conclusion}

This work reported the design and simulations of a novel OP-AMP scheme capable of driving a large capacitive load implemented in 1um FDSOI technology. The biasing circuitry eliminates the need for an extra large circuitry allowing saving in power consumption without deteriorating the gain bandwidth. From the presented results summarized in table 1, this low power OP-AMP achieves a good stability, higher gain and lower power dissipation with an optimized chip size.

\section{References:}

[1] J Karki - Mixed Signal and Analog Operational Amplifiers -April 1998 Digital Signal Processing Solutions " Understanding Operational Amplifier Specifications" 
[2] Hitesh Modi, Nilesh D. Patel, "Design and Simulation of two Stage OTA using $0.18 \mathrm{pm}$ and 0.35 p A.D. Grasso, G. Palumbo Fellow, Pennisi Fellow, "Dual push-pull high-speed rail-to-rail CMOS buffer amplifier for flatpanel displays" IEEE Transactions on Circuits and Systems II: Express Briefs, 2018

[3] OP-AMPAND ITSAPPLICATIONS https://kobita1234.files.wordpress.com/2016/11 /ch-68.pdf

[4] Phillip E. Allen and Douglas R. Holberg, CMOS Analog Circuit Design, Saunders College Publishing HBJ, 2002.

[5] H. Ameziane,H.Akhamal, K. Zared, H. Qjidaa "Full On-chip low dropout voltage regulator with an enhanced transient response for low power systems" International Journal of Electrical and Computer Engineering (IJECE) Vol 9, No 6: December 2019.

[6] Using op amps with data converters https://www.analog.com/media/en/trainingseminars/design-handbooks/Op-AmpApplications/Section3.pdf

[7] Himadri Singh Raghav, B.P. Singh, Sachin Maheshwari, "Design of Low Voltage OTA for Bio-medical Application", IEEE International Conference on Microelectronics, Communication and Renewable Energy, pp. 2013.
[8] Eggermont, J.-P.; De Ceuster, D.; Flandre, D.; Gentinne, B.; Jespers, P.G.A.; Colinge, J.-P. Design of SOI CMOS operational amplifiers for applications up to $300{ }^{\circ} \mathrm{C}$. IEEE J. SolidState Circuits 1996, 31 (2), 179-186.

[9] Silveira, F.; Flandre, D.; Jespers, P.G.A. A gm/Id based methodology for the design of CMOS analog circuits and its application to the synthesis of a silicon-on-insulator micropower OTA. IEEE J. Solid-State Circuits 1996, 31 (9), 1314-1319.

[10] B. Razavi, Design of Analog CMOS Integrated Circuits, McGraw-Hill Book Company, 2001

[11] A.D. Grasso, G. Palumbo Fellow, Pennisi Fellow, "Dual push-pull high-speed rail-to-rail CMOS buffer amplifier for flat-panel displays" IEEE Transactions on Circuits and Systems II: Express Briefs, 2018

[12] K. H. Mak, M. W. Lau, J. Guo, T. W. Mui, W. L. Goh and L. N. Leung, "A 0.7V 24 $\mu$ A Hybrid OTA Driving $15 \mathrm{nF}$ Capacitive Load with 1.46MHz GBW," IEEE J. Solid State Circuits, vol.50, no.11, pp. 2750-2757, Nov. 2015. 


\section{Creative Commons Attribution License 4.0 (Attribution 4.0 International, CC BY 4.0)}

This article is published under the terms of the Creative Commons Attribution License 4.0

https://creativecommons.org/licenses/by/4.0/deed.en US 